\title{
Sobre cómo dos teorias clásicas pueden (o no) resolver el pro- blema de ciertos enunciados pragmáticamente extraños
}

Carlota Piedehierro Saez

Gerlhis (Groupe d'Études et de Recherches en Linguistique Hispanique)

Université Sorbonne Nouvelle -Paris 3

Centre Censier 13, rue de Santeuil - 75231 Paris Cedex 05

E-mail : carpisa1702@hotmail.com

\section{SOBRE CÓMO DOS TEORÍAS CLÁ- SICAS PUEDEN (O NO) RESOLVER EL PROBLEMA DE CIERTOS ENUNCIADOS MENTE EXTRAÑOS PRAGMÁTICA-}

RESUMEN: Este artículo tiene dos objetivos: en primer lugar, busca delimitar las diferencias que separan a la Teoría de la relevancia y a la Pragmática integrada comparando la distinta concepción que en ambos enfoques se tiene sobre los conceptos frase-enunciado o instrucción, a menudo utilizados de forma amalgamada en el análisis de enunciados donde aparecen marcadores discursivos. En segundo lugar, pretende mostrar que una de esas dos teorías puede ser más eficaz para explicar la inaceptabilidad de ciertas secuencias pragmáticamente extrañas. Se ilustra este postulado con el análisis de varios enunciados donde aparecen marcadores discursivos utilizados de forma afectada. Sirviéndonos de la división entre estructura super ficial y estructura profunda, se busca aquí apelar a la necesidad de recuperar ideas básicas del enfoque sintagmático de Anscombre y Ducrot que no han sido a nuestro juicio suficientemente desarrolladas en los estudios sobre marcadores del discurso en el ámbito hispánico.

PALABRAS CLAVES: teoría de la relevancia; pragmática integrada; frase-enunciado; instrucción; marcadores discursivos.

SUMARIO: 1. Introducción. 2. La Teoría de la relevancia y la Pragmática integrada: qué las une, qué las separa. 2. 1. Dos formas de definir lo implícito. 2. 2. El par frase-enunciado. 2. 3. Dos respuestas para dos preguntas. 2. 4. El concepto de ins trucción. 3. Aplicación al análisis de enunciados pragmáticamente extraños. 4. Conclusiones.

\section{CAN TWO CLASSICAL LIN- GUISTIC THEORIES EXPLAIN CERTAIN PRAGMATICALLY STRANGE UTTERANCES?}

ABSTRACT: The aim of this paper is twofold. Firstly, it attempts to delimit the differences between Relevance Theory and Integrated Pragmatics, comparing their conceptualization of the concepts sentence-utterance and instruction, usually confused in the analysis of utterances presenting discourse markers. Secondly, it aims at demonstrating that one of the two approaches explains more efficiently the unacceptability of certain pragmatically strange sequences. To do so, various statements are examined in which discourse markers are used affectedly. This paper, based on the distinction between deep and surface structure, points out the need to recover the basic ideas of Anscombre and Ducrot's syntagmatic approach, which have not been sufficiently developed in the studies concerning discourse markers in Spanish.

KEY WORDS: relevance theory; integrated pragmatics; sentence-utterance; instruction; discourse markers.

SUMMARY: 1 . Introduction. 2. The Relevance Theory and Integrated Pragmatics: what they share and what sets them apart. 2.1. Two ways to define what is implicit. 2 .2. The pair sentenceutterance. 2.3 Two answers to two questions. 2.4 The concept of instruction. 3. Application to the analysis of pragmatically strange utterances. 4. Conclusions.

\section{COMMENT DEUX THEORIES CLASSIQUES PEUVENT (OU PAS) RESOUDRE LE PROBLEME DE CERTAINS ENONCES PRAGMATIQUEMENT INACCEPTABLES}

RÉSUMÉ: Le but de ce travail est double: dans un premier temps, il cherche à délimiter les différences qui séparent la Théorie de la pertinence et la Pragmatique intégrée en comparant comment les concepts phrase-énoncé ou instruction (souvent confondus dans l'analyse d'énoncés comportant des marqueurs discursifs) sont définis dans chaque approche. Dans un deuxième temps, il tente de montrer qu'une de ces deux théories peut être plus efficace pour expliquer la nonacceptabilité de certaines séquences qui se révèlent bizarres dans un point de vue pragmatique. Pour illustrer cette hypothèse, une analyse de plusieurs énoncés incluant des marqueurs discursifs utilisés de façon incorrecte est proposée. En nous servant de la distinction entre structure superficielle et structure profonde, nous cherchons à montrer qu'il est nécessaire de reprendre certaines idées essentielles de l'approche syntagmatique d'Anscombre et Ducrot, idées selon nous insuffisamment développées dans les études sur les marqueurs discursifs dans le monde hispanique.

MOTS CLÉS: théorie de la pertinence; pragmatique intégrée; phrase-énoncé instruction; marqueurs discursifs.

SOMMAIRE: 1. Introduction. 2. La Théorie de la pertinence et la Pragmatique intégrée: qu'est-ce qu'elles partagent et qu'est-ce qui les sépare ? 2. 1. Deux manières de définir l'implicite. 2. 2. Le couple phraseénoncé. 2. 3. Deux réponses pour deux questions. 2. 4. Le concept d'instruction. 3. Application à l'analyse d'énoncés pragmatiquement inacceptables. 4. Conclusions.
Fecha de Recepción Fecha de Revisión Fecha de Aceptación Fecha de Publicación
$02 / 04 / 2016$

$21 / 08 / 2018$

$11 / 09 / 2018$

$01 / 12 / 2018$ 


\section{Sobre cómo dos teorias clásicas pueden (o no) resolver el pro- blema de ciertos enunciados pragmáticamente extraños}

\section{CARlota PiedeHIERRO SÁEZ}

\section{INTRODUCCIÓN}

La Teoría de la relevancia (Sperber y Wilson, 1986) y la Pragmática integrada (Anscombre y Ducrot, 1976, 1983) son hoy consideradas dos enfoques clásicos en el análisis de la interpretación de los enunciados. Como otras teorias pragmáticas, su gran aportación tiene que ver sobre todo con que ambas suponen una superación de la Teoría del código, rebasada al poner en evidencia que, en lengua, lo implícito es, al menos, igual de importante que lo explícito. La influencia de sus postulados ha sido muy relevante en los estudios lingüísticos de las últimas décadas. En lo que concierne al ámbito del español, su peso ha sido muy notable, especialmente en los estudios dedicados al análisis de secuencias que incluyen marcadores del discurso. Precisamente en este campo concreto, las investigaciones de los últimos tiempos se decantan por enfoques de naturaleza integral, donde la interpretación de enunciados que incluyen marcadores discursivos se analiza utilizando herramientas tomadas de diferentes teorias ${ }^{1}$.

A pesar de que consideramos que un enfoque integral puede aportar las mayores ventajas en el estudio de la interpretación de enunciados que incluyen marcadores del discurso, este tipo de proceder no debe hacernos olvidar que los conceptos y herramientas de cada teoría se corresponden con un determinado marco teórico y que es necesario utilizarlos teniendo en cuenta este hecho. Con el objetivo de incidir en esta idea, en este trabajo intentamos desglosar las diferencias que separan a la Teoria de la relevancia y a la Pragmática integrada en cuanto a sus objetivos de análisis y en cuanto a la operacionalización de ciertos conceptos que, si bien son comunes a ambas teorias, responden en realidad a cosas muy distintas. Nuestro objetivo con ello no es solamente subrayar estas diferencias sino también llamar la atención sobre el hecho de que ciertos constructos de la Pragmática integrada de Anscombre y Ducrot no han sido suficientemente desarrollados en los estudios sobre los marcadores discursivos del español y pueden ser retomados para resolver eficazmente problemas como los que se dan en enunciados que resultan pragmáticamente extraños.

\section{La Teoría de la Relevancia y la PRAgmática integrada: QUÉ las une, QUÉ LAS SEPARA}

\footnotetext{
1 Los enfoques eclécticos constituyen una tendencia hoy generalizada en el estudio de los marcadores del discurso en español. Véase, a este respecto, muchos trabajos de Portolés (Portolés, 1989, 1993, 1995a, 1995b, 1998a, 1998b, 1999, 2000a, 2000b, 2001 [1998], 2004a, 2004b), o de Cortés y Camacho (2005), el compendio coordinado por Óscar Loureda Lamas y Esperanza Acín Villa (2010), la tesis de Llopis Cardona (2011) o la de Piedehierro (2012).
} 


\subsection{DOS FORMAS DE DEFINIR LO IMPLÍCITO}

Tanto la Teoría de la relevancia como la Pragmática integrada son dos perspectivas que estudian la interpretación de los enunciados que emiten los hablantes. Como punto de partida, las dos están de acuerdo en considerar que la Teoría del código es una teoría fallida para estudiar la interpretación de los enunciados pues, en lengua, no todo es explícito:

\footnotetext{
Dire que les langues naturelles sont des codes, destinés à la transmission de l'information d'un individu à un autre, c'est admettre du même coup que tous les contenus exprimés grâce à elles sont exprimés de façon explicite. Par définition en effet, une information encodée, c'est, pour celui qui sait déchiffrer le code, une information manifeste, une information qui se donne comme telle, qui s'avance, qui s'étale. Ce qui est dit dans le code est totalement dit, ou n'est pas dit du tout. (Ducrot, 1991 [1972]: 5)

[...] le modèle du code n'est pas le seul à expliquer en principe comment la communication est possible. En effet, la communication a également été décrite comme un processus inférentiel de reconnaissance des intentions du locuteur. (Sperber y Wilson, 1989 [1986]: 21)
}

Hay sin embargo algo fundamental que las separa: lo implícito no está, para las dos perspectivas, en el mismo lugar. Para la pragmática relevantista, la interpretación es fruto de un proceso inferencial que tiene lugar gracias a la mediación entre los signos y el contexto; para la Pragmática integrada, por el contrario, la interpretación está anclada en las estructuras lingüísticas mismas. En otras palabras, la primera pretende responder a la cuestión de qué interpretamos a partir de lo que nos dicen mientras que la segunda busca saber qué características tiene lo que decimos que explican que, a partir de ello, interpretemos lo que interpretamos.

Estas dos preguntas explican por qué el modelo del código tiene inconvenientes distintos según cada teoria: para la pragmática relevantista el esquema de la comunicación basado en el código no es operativo porque no da cuenta de lo que ocurre verdaderamente en la comunicación:

Il est vrai qu'une langue est un code qui associe des représentations phonétiques à des représentations sémantiques. Mais la représentation sémantique d'une phrase est loin de coïncider avec les pensées qui peuvent être communiquées en énonçant cette phrase. On passe de la représentation sémantique à la pensée communiquée non par un surcroît de codage, mais au moyen d'inférences. (Sperber y Wilson, 1989 [1986]: 21)

En un intercambio comunicativo, lo que un hablante quiere decir es mucho más de lo que expresa en lo que dice. Asumiendo la idea de Grice (1975) según la cual la representación semántica no es suficiente para dar cuenta de la interpretación de los enunciados, la Teoría de la relevancia asume que para aprehender la intención del locutor de un enunciado es necesario un proceso inferencial que recupere las intenciones del hablante. Por el contrario, para la Pragmática integrada el modelo del código es problemático no 
por el hecho de que no sea capaz de dar cuenta de cómo funciona la comunicación sino porque implica de modo ineluctable que la función principal de las lenguas es comunicar, entendiendo esta función como la transmisión de información:

[...] si une linguistique de la communication doit nécessairement faire entrer dans sa description de l'acte de parole la dualité des interlocuteurs, il arrive souvent qu'on restreigne le sens du mot "communication", en le forçant à désigner seulement un type particulier de relation intersubjective, la transmission de l'information. Communiquer, ce serait, avant toute chose, faire savoir, mettre l'interlocuteur en possession de connaissances dont il ne disposait pas auparavant : il n'y aurait communication que lorsque, et dans la mesure où, il y a communication de quelque chose. Cette conception de la communication transparaît lorsqu'on compare la langue à un code. (Ducrot, 1991 [1972]: 2)

Esta diferencia entre las dos posturas es fundamental pues, aunque las dos parten del mismo punto, este no está motivado por el mismo problema. Como consecuencia, los objetivos de análisis y las herramientas de trabajo de las dos son diferentes y, aunque en muchas ocasiones utilicen los mismos conceptos, estos no reflejan las mismas herramientas de análisis ni estas tienen las mismas funciones. En los apartados siguientes nos proponemos dar cuenta de las diferencias que median entre las dos teorias en lo que se refiere a varias cuestiones: en primer lugar, el par frase-enunciado; en segundo lugar, el diferente enfoque metodológico de las dos perspectivas; finalmente, la muy diferente concepción que se tiene del concepto de instrucción, a menudo considerado como equivalente pero en realidad con una función muy alejada en cuanto a su alcance y su operatividad dentro de cada modelo.

\subsection{EL PAR FRASE-ENUNCIADO}

La Teoria de la relevancia entiende que la diferencia entre frase y enunciado es fundamental, pero lo es en el sentido de que corresponde a la primera ser la manifestación de la representación semántica mientras que corresponde al segundo ser la verdadera entidad portadora de los pensamientos comunicados (es decir, de la dimensión pragmática):

La représentation sémantique d'une phrase correspond en quelque sorte au noyau de sens qui est commun à tous les énoncés de la phrase en question. Mais différents énoncés d'une même phrase peuvent avoir -et, en général, ont- différentes interprétations. L'étude de la représentation sémantique des phrases relève de la grammaire ; l'étude de l'interprétation des énoncés relève de ce que l'on appelle aujourd'hui la pragmatique. (Sperber y Wilson, 1989 [1986]: 22-23)

Por su parte, la diferencia entre frase y enunciado para la Pragmática integrada no tiene que ver con que la primera es una especie de representación abstracta de todas las posibles manifestaciones que pueden darse en forma del segundo (i.e. el enunciado). Para empezar, porque hay un asunto previo fundamental: el significar de la frase y el del enunciado son distintos: 
la primera tiene significación, mientras que el segundo tiene sentido y ello no solo implica un diferente estatuto metodológico sino una distinta naturaleza en las dos dimensiones:

Cuando sea cuestión de caracterizar semánticamente a una frase hablaré de "significación", y reservaré la palabra "sentido" para la caracterización semántica del enunciado. Entre el sentido y la significación hay para mí a la vez una diferencia de estatuto metodológico y una diferencia de naturaleza. De estatuto metodológico porque, en el trabajo del lingüista semántico, el sentido pertenece al dominio de lo observable, al dominio de los hechos: el hecho que tenemos que explicar es que tal enunciado tenga tal(es) sentido(s), es decir, que sea susceptible de tal(es) interpretación(es). (Ducrot, 1986 [1984]: 184)

Para seguir, porque esta diferencia implica ir más allá del hecho de relacionar la significación con el nivel léxico-gramatical y el sentido con el nivel pragmático:

Para dar cuenta de manera sistemática de la asociación "observada" entre sentidos y enunciados, elijo asociar a las frases realizadas por los enunciados un objeto teórico etiquetado como "significación". La maniobra me parece interesante en la medida en que supongo posible formular leyes, de un lado para calcular la significación de las frases a partir de su estructura léxico-gramatical y, del otro, para prever, a partir de esta significación, el sentido de los enunciados.

Pero más allá de esta diferencia metodológica, yo planteo entre el sentido y la significación una diferencia de naturaleza. Con ello aspiro a rebatir la concepción habitual según la cual el sentido del enunciado es la significación de la frase salpimentada con algunos ingredientes tomados de la situación de discurso. Según esta concepción, el sentido incluiría por un lado la significación y, por el otro, los añadidos aportados por la situación. Personalmente me niego a considerar la significación como una parte del sentido [...]. Prefiero representar a la significación como un conjunto de instrucciones dadas a las personas que tienen que interpretar los enunciados de la frase, instrucciones que establecen las maniobras que se han de realizar para asociar un sentido a estos enunciados. (Ducrot, 1986 [1984]: 185)

Para la Pragmática integrada, el sentido del enunciado no se obtiene a partir de la representación semántica de la frase a la cual se le añaden las circunstancias de la enunciación sino que parte de la significación, la cual es vista como un conjunto de instrucciones dadas al interlocutor. ¿Qué significa esto? Que el análisis de la frase no se establece a partir de condiciones de verdad, visión que la Teoría de la relevancia sí defiende (al menos para el análisis de una parte de los signos lingüísticos):

Ici, la distinction entre une phrase et un énoncé est capitale. Un énoncé possède un ensemble de propriétés, les unes linguistiques, les autres non. [...] Une grammaire générative fait abstraction de toutes les propriétés d'un énoncé qui ne sont pas purement linguistiques et décrit une structure linguistique abstraite, la phrase. Cette structure linguistique peut être commune à une multitude d'énoncés que seules leurs propriétés extralinguistiques distinguent les uns des autres. Par définition, la représentation sémantique qu'une grammaire générative assigne à une phrase ne tient pas compte des propriétés extralinguistiques des énoncés de cette phrase, comme par exemple le moment et le lieu de l'énonciation, l'identité du locuteur, ou ses intentions. (Sperber y Wilson, 1989 [1986]: 22-23) 


\subsection{DOS RESPUESTAS PARA DOS PREGUNTAS}

Sin duda ello supone que el tipo de análisis que puede abarcar una teoría no puede ser abarcado por la otra y viceversa. Ello explica, por ejemplo, la crítica que Diane Blakemore (2002) lanza a la imposibilidad de la Pragmática integrada para dar cuenta de las interpretaciones de los enunciados que están directamente determinadas por el contexto:

$[\ldots]$ argumentation theory is unable to address the sort of questions raised in this chapter, namely, how a hearer is able to recover the intended interpretation for context dependent expressions. (Blakemore, 2002: 27-28)

Para la autora relevantista, esta limitación de la perspectiva pragmática desarrollada por Anscombre y Ducrot se debe a que en su teoría estos autores no ven un enunciado como un producto de un hablante particular con unas intenciones particulares, sino como una colección de diferentes puntos de vista, "[a]nd a point of view is not anchored in the mind of a particular person with particular thoughts" (Blakemore, 2002: 27). Desde la perspectiva de la Teoría de la relevancia, el hecho de que la Teoría de la Argumentación estudie los enunciados como entidades ajenas a un fenómeno cognitivo impide explicar ciertas interpretaciones que solo pueden ser aprehendidas vía el contexto. Y dichas interpretaciones son posibles gracias a las instrucciones de procesamiento que actúan bajo la acción del contexto ${ }^{2}$. Pongamos un ejemplo. Si tomamos un enunciado como (1):

(1) "Ramón ha vuelto a perder la carpeta roja".

Podemos lanzar las hipótesis de que este puede provocar los siguientes supuestos ${ }^{3}$ :

\section{Supuestos:}

a. Ramón es un olvidadizo (una constatación).

b. Ramón es un incompetente (un reproche).

c. Hoy tampoco podremos celebrar la reunión (una preocupación/una queja).

Desde el punto de vista de la pragmática relevantista, las distintas interpretaciones que pueden atribuirse al enunciado (1) no pueden obtenerse a partir de los signos que lo constituyen y hay que servirse de otros elementos para reconstruirlas: se asume que dicho enunciado muestra (hace osten-

\footnotetext{
2 Para la Pragmática integrada la enunciación no es un fenómeno fisiológico llevado a cabo por un individuo, sino la manifestación más clara del carácter autorreferencial de la lengua: un enunciado es la mostración de su propia enunciación.

${ }^{3}$ Un supuesto es un pensamiento procesado que se obtiene después de aplicar explicaturas a una forma proposicional.
} 
siva) una variedad de hipótesis y el auditor se fija en aquella(s) que le parece(n) relevante(s). El principio de relevancia permite al receptor reconocer la intención informativa del locutor, la cual es, además, mutuamente manifiesta tanto para el oyente como para el locutor. Así, partiendo de:

Enunciado: Ramón ha vuelto a perder la carpeta roja.

por descodificación, obtenemos la forma lógica:

Forma lógica: [Ramón-perder-la-carpeta-roja-ahora-y-antes-de-ahora]

que puede dar lugar a las siguientes explicaturas:

\section{Explicaturas:}

(a) Es un hecho que Ramón ha vuelto a perder la carpeta roja.

(b) El incompetente de Ramón ha vuelto a perder la carpeta roja.

(c) Es un problema que Ramón haya vuelto a perder la carpeta roja.

Tras el enriquecimiento contextual, se obtienen las siguientes interpretaciones:

Interpretaciones (implicaturas):

(a) Ramón es un despistado (una constatación).

(b) Ramón es un incompetente (un reproche).

(c) Hoy tampoco podremos celebrar la reunión (una preocupación/una queja).

De modo que, según este proceso, la emisión de (1) implica la aparición de un conjunto de supuestos y el interlocutor, gracias a la puesta en marcha de su contexto cognitivo, elige el supuesto que para él es más relevante. El procedimiento desde la Pragmática integrada es diferente: a esta perspectiva no le interesa llegar a decir cuál de las interpretaciones de (1) es la que recoge la verdadera intención del emisor, pues lo que le interesa es explicar por qué el ejemplo (1) puede tener las interpretaciones (a), (b) o (c). En el fondo, lo que ocurre es que las dos teorias responden a preguntas distintas. La Teoría de la relevancia busca responder a la pregunta de cuál de entre todas las interpretaciones que puede tener un enunciado es la que pretende emitir el locutor; la Pragmática integrada busca por su parte responder a la pregunta de por qué un enunciado como (1) puede tener las interpretaciones que tiene. De este modo, la Pragmática integrada puede ver, como la Teoría de la relevancia, las mismas interpretaciones para el ejemplo (1) -una constatación, un reproche o una preocupación-, pero no llega a estos significados después de que el enunciado sea emitido en un intercambio comunicativo, sino que lo busca en la propia información que las palabras aportan sobre sí mismas y sobre su modo de aparecer en el discurso. En la concepción de 
Anscombre y Ducrot, la disposición, el significado y el funcionamiento de las palabras del enunciado "Ramón ha vuelto a perder la carpeta roja" explican que este pueda ser visto como una constatación, un reproche o una queja.

\subsection{EL CONCEPTO DE INSTRUCCIÓN}

Como es sabido, el concepto instrucción en la Teoría de la relevancia se aplica al análisis semántico de palabras como los marcadores del discurso porque se considera que este tipo de elementos poseen un tipo de significado que es denominado procedimental o de procesamiento, el cual es diferente del denominado conceptual: mientras este se refiere a la información léxica sobre el contenido proposicional de los enunciados, aquel hace referencia a la información sobre cómo procesarlo. Decir que los marcadores son palabras que poseen un significado instruccional equivale desde este punto de vista a definirlos como palabras que no contribuyen a las condiciones de verdad de los enunciados donde aparecen, sino que dan información sobre cómo procesar el contenido que aparece en ellos. Esta diferencia, enunciada por primera vez en Blakemore $(1987)^{4}$, ha sido objeto de varias modificaciones, pues se ha ido viendo que considerar que el significado de los marcadores es meramente procedimental da lugar a varios problemas. El primero de ellos tiene que ver con pretender definir el significado de los marcadoresa través de una única instrucción de procesamiento. Esta idea, que llevó a Blakemore (1992) a proponer una clasificación general de los marcadores del inglés utilizando como criterio los diferentes tipos de efectos contextuales a los que daban lugar, tiene el inconveniente, como señala Murillo (2010: 249), de que no ofrece "las herramientas para el estudio de las diferencias entre los elementos próximos pero no intercambiables en todos sus usos (but, however y nevertheless, por ejemplo)". Para resolver este problema, Blakemore ha propuesto extender la noción de significado procedimental y no restringirla a la codificación de efectos cognitivos, añadiendo a la definición semántica ciertas restricciones contextuales. Sin embargo, como explica Murillo (2010), esta solución tampoco resuelve todas las limitaciones que supone la defensa de una única instrucción aplicada a cada marcador. Un segundo problema asociado al concepto de significado procedimental ha tenido que ver, como también es sabido, con la asociación de este con el significado no vericondicional, defendida en los primeros trabajos de Blakemore. Sperber y Wilson (1990) reaccionaron a esta asociación en un artículo clásico de la teoría donde exponían que la identificación del binomio conceptual-procedimental con vericondicional-no vericondicional no debía sostenerse. Trabajos recientes dentro de la teoria se distancian cada vez más de esta asociación (hasta el punto de que actualmente "se sostiene que la dis-

\footnotetext{
${ }^{4}$ Recuérdese que Blakemore (1987) partió de la noción de Grice (1975) de implicaturas convencionales para hacer su división entre significado conceptual y procedimental.
} 
tinción entre significado vericondicional y no vericondicional no es fundamental", Murillo 2010: 252) pero se trata no obstante de una cuestión que sigue quedando irresuelta dentro de una perspectiva que defiende, como base para el estudio semántico, la idea de que las palabras de las lenguas naturales codifican significados de distinta naturaleza. Un tercer y último problema tiene que ver con la limitación de entender el significado procedimental como un constructo de naturaleza cognitiva. Como ha sido señalado en varios trabajos (Portolés, 2001 [1998]; Pons, 2004), este enfoque, si bien es coherente con los postulados básicos de la teoria, se muestra muy limitado para dar cuenta de ciertos aspectos lingüísticos que no pueden dejarse de lado a la hora de analizar elementos como los marcadores del discurso.

Para resolver los problemas relacionados con estas y otras limitaciones de la Teoría de la relevancia, en el estudio de los marcadores del discurso dentro del ámbito hispánico se ha propuesto y se ha desarrollado un enfoque ecléctico, integrador de varios puntos de vista, donde se toman ideas de varias perspectivas y se aplican al análisis de este tipo de unidades ${ }^{5}$. Aunque nosotros también defendemos la necesidad de un enfoque integral para afrontar los diferentes problemas que atañen al análisis de los marcadores del discurso, creemos que se puede ir más allá para superar las limitaciones que presenta la Teoría de la relevancia. Quisiéramos defender aquí que puede haber una solución diferente a las limitaciones que presenta este enfoque: frente a la ampliación del tipo de instrucciones o a su división en diferentes subtipos, nuestra idea es reconsiderar el concepto mismo de instrucción. Y para ello proponemos recuperar la concepción instruccional primigenia de los trabajos de la Pragmática integrada desarrollada por Anscombre y Ducrot.

Si para la Teoría de la relevancia una instrucción es una guía que dirige al interlocutor para que este infiera la intención del enunciado del locutor, para la Pragmática integrada, en cambio, una instrucción es una consigna lingüística acatada por una palabra que, junto con otras palabras, conforma una frase de una lengua, comprometiéndola a ser susceptible de mostrar una determinada significación y, como consecuencia, de explicar el sentido de un enunciado. Como consecuencia, las instrucciones no están para cada una de las teorias en el mismo lugar; en concreto, si para los relevantistas se sitúan en la parte inferencial del proceso comunicativo, para los autores de la Argumentación en la lengua se sitúan en una parte no explícita del análisis lingüístico que habria de ser localizada en una hipotética estructura profunda, allá donde se ubican las frases, diferentes de los enunciados:

J'entendrai par phrase [...] une entité linguistique abstraite, purement théorique, en l'occurrence un ensemble de mots combinés selon les règles de la syntaxe, ensemble pris hors de toute situation de discours; ce que produit un locuteur, ce qu'entend un auditeur, ce n'est donc pas une phrase, mais un énoncé particulier d'une phrase. (Ducrot et al., 1980: 7)

\footnotetext{
5 Véase nota 1.
} 
La división en la Pragmática integrada entre estructura superficial y estructura profunda responde al reparto entre, por un lado, enunciado-frase y, por el otro, hipótesis externas-hipótesis internas. Las primeras representan los “observables", es decir, los hechos que, bajo decisión del lingüista, constituirán su objeto de análisis; las segundas representan los mecanismos que sirven para explicar las primeras:

Nous entendons par description sémantique le couple hypothèses externes/hypothèses internes (cf. O. Ducrot, 1972, p. 120). [...]

La première catégorie, celle des hypothèses externes, a pour fonction de fournir ce que l'on convient d'appeler les faits, ou encore les données, les observables. Quels sont ces faits dont s'occupe la description sémantique ? D'une part le découpage d'une occurrence de discours en segments discursifs élémentaires -que nous appellerons plus loin énoncés -d'autre part l'acceptabilité ou la non-acceptabilité de telle ou telle occurrence effective, ainsi que l'attribution d'un sens à ces occurrences et la détermination des relations sémantiques qu'elles entretiennent entre elles. Il ne s'agit dans aucun de ces cas d'un donné brut imposé de l'extérieur au linguiste, mais d'un ensemble de décisions de sa part : il est coextensif à toute description linguistique de créer ses propres observations. [...] Ces mécanismes explicatifs impliquent des hypothèses d'une toute autre nature. Ce sont les hypothèses internes. Elles consistent à imaginer des entités abstraites, à les mettre en correspondance avec les observables, et à construire un appareil formel permettant de calculer -entre entités abstraites- des rapports analogues à ceux postulés entre les observables correspondants. (Anscombre y Ducrot, 1978: 348)

Las instrucciones en la Pragmática integrada son hipótesis internas: son un mecanismo construido por el lingüista cuya función es explicar los datos que quieren ser analizados (en concreto, la interpretación de un enunciado). Las instrucciones son, de este modo, un constructo teórico, de naturaleza completamente diferente al que se maneja en la Teoría de la relevancia. Para empezar, porque al localizarse en la estructura profunda, las instrucciones de la Pragmática integrada se sitúan en el nivel de la frase (y no el enunciado); para seguir, porque la frase tiene significación, y la significación no es algo "comunicable":

[...] ce que nous entendons par signification (du mot ou de la phrase) est tout autre chose que le "sens littéral " dont il vient d'être question. Car elle n'est pas un constituant du sens de l'énoncé, mais lui est au contraire complètement hétérogène. Elle contient surtout, selon nous, des instructions données à ceux qui devront interpréter un énoncé de la phrase, leur demandant de chercher dans la situation de discours tel ou tel type d'information et de l'utiliser de telle ou telle manière pour reconstruire le sens visé par le locuteur. Si l'on admet cette conception, on doit reconnaître que la signification d'une phrase n'est pas quelque chose de communicable, qu'elle n'est pas quelque chose qui puisse se dire : on arrive, tant bien que mal, à se faire comprendre avec des énoncés : il n'est même pas question d'essayer de se faire comprendre avec des phrases. (Ducrot et al., 1980:12)

Las instrucciones, por tanto, desde la Pragmática integrada, no son algo que sea "procesado" cognitivamente. Y ello es así porque no sirven para guiar 
las inferencias comunicadas en los enunciados sino que en realidad constituyen el núcleo semántico de las frases y sirven para establecer "las maniobras que se han de realizar para asociar un sentido a [los] enunciados" (Ducrot, 1986 [1984]: 185). ¿Por qué las instrucciones pertenecen a la frase y no al enunciado?, es más, ¿por qué es necesario distinguir entre frase y enunciado como dos entidades de distinta naturaleza? Porque es necesario hacer esta división terminológica para poder responder a la pregunta que busca responder la Pragmática integrada, recordemos: "[a]ttribuer un sens à un énoncé, c'est entreprendre une démarche explicative, c'est chercher pourquoi l'énoncé a été produit" (Ducrot et al. 1980: 22).

\section{APLICACIÓn AL ANÁlisis de ENUNCIAdOS PRAGMÁticAMENTE EXTRAÑos}

Para poder saber por qué un enunciado ha sido producido o, dicho de otro modo, para poder saber cuál es la intención de un locutor cuando emite un enunciado, ha de saberse primero por qué un enunciado dado puede tener las interpretaciones que tiene. Para ello, este ha de ser estudiado no partiendo de lo que el locutor produce, es decir, no partiendo de lo que su interlocutor entiende, sino tomando un ente lingüístico abstracto, puramente teórico, un conjunto de palabras combinadas según las reglas de la gramática de una lengua: una frase. Solo sabiendo cuál es la significación de una frase puede llegar a saberse cuál es el sentido del enunciado. La significación de la frase (o de las palabras que la constituyen) está formada por instrucciones, es decir, un conjunto de reglas y/o advertencias "données à ceux qui devront interpréter un énoncé de la phrase, leur demandant de chercher dans la situation de discours tel ou tel type d'information et de l'utiliser de telle ou telle manière pour reconstruire le sens visé par le locuteur" (Ducrot et al., $1980: 12$ ). Nuestra idea es que este enfoque no solo es más pertinente para dar cuenta de por qué un enunciado puede producir ciertas interpretaciones sino, también, para explicar por qué hay secuencias que conducen a interpretaciones fallidas. Para ilustrar este hecho, nos proponemos ahora exponer el análisis de varios enunciados que, desde el punto de vista pragmático, resultan inaceptables. Se trata de los siguientes:

(2) \# "No fue ayer cuando llegó el paquete, pero anteayer".

(3) \# "Tienes que irte; en efecto, es muy peligroso que te quedes".

(4) \# "Juan terminó el trabajo, pues se fue".

No buscamos hacer aquí un estudio exhaustivo de todas las instrucciones implicadas en estos tres enunciados, sino dar cuenta solamente de aquellas que resultan fundamentales para explicar su no aceptabilidad. Para ello, tendremos en cuenta dos consideraciones: en primer lugar, que es nuestra decisión, como lingüistas, considerar que los enunciados que vamos a analizar son inaceptables desde el punto de vista pragmático. Este hecho constituye una de nuestras hipótesis externas. En segundo lugar, que 
nuestro objetivo será intentar descubrir qué reglas, de las que se supone deben ser acometidas por los marcadores objeto de estudio, se están incumpliendo en cada uno de los enunciados analizados. Este procedimiento constituirá nuestras hipótesis internas. Nuestro análisis partirá de la consideración de que es necesario, para ello, distinguir entre estructura superficial y estructura profunda. Esta decisión tiene como veremos varias ventajas: permite, por ejemplo, recuperar segmentos implícitos que se sobrentienden en el contexto lingüístico y que son fundamentales para explicar ciertos fenómenos o dar cuenta de qué parte del contenido de un miembro del discurso representa el verdadero alcance de un marcador. Empezaremos con el estudio de pero en el ejemplo (2).

Según la Pragmática integrada, para estudiar la significación de una palabra hay que mostrar que esta significación, en colaboración con la asignada a las demás palabras de la frase, contribuirá a explicar el sentido dado al enunciado:

[...] pour fonder la caractérisation sémantique d'un mot, pour se justifier de lui attribuer telle ou telle signification, il faut d'abord montrer que cette signification, coopérant avec celle attribuée aux autres mots d'une phrase, permet de prévoir la signification de la phrase, qui, elle-même [...] n'a d'autre fonction que d'expliquer, compte tenu des conditions particulières d'emploi, le sens de l'énoncé. (Ducrot et al., 1980: 9).

En el caso concreto de palabras como los marcadores del discurso, esta idea implica que en su estudio ha de analizarse no solo el marcador en cuestión sino además las estructuras que se encuentran a su izquierda y/o a su derecha:

Que l'on étudie mais, eh bien, décidément ou d'ailleurs, on se trouve en présence de mots dont le rôle habituel est d'établir un lien entre deux entités sémantiques. C'est pourquoi nous ne pouvons pas décrire mais ou d'ailleurs isolément : nous devons décrire les structures $P$ mais $Q, P$ d'ailleurs $Q$. On se trouve donc d'emblée devant le problème de déterminer ces $\mathrm{P}$ et ces $\mathrm{Q}$ sur lesquels portent les morphèmes étudiés. (Ducrot et al., 1980: 22).

Partiendo de esta consideración, no podemos estudiar el valor semántico de pero en (2) sin tener en cuenta lo que se encuentra a su izquierda y a su derecha. Al estudiar cómo un marcador se combina con las demás palabras para definir la significación de la frase, se está no solo intentando explicar por qué el enunciado correspondiente a dicha frase tendrá el sentido que tiene sino también cómo se ha llegado a él: las características de lo que rodea al marcador determinan por qué este convoca las instrucciones que convoca para que el interlocutor interprete como interpreta el sentido del enunciado.

Como se sabe, pero, considerada conjunción coordinante adversativa en la gramática clásica, es hoy definida como un conector al que, desde la perspectiva ecléctica a la que aludiamos antes, se le pueden aplicar diversas instrucciones: se trata, en primer lugar, de un signo que presenta una "restricción" a una información anterior; en segundo lugar, y tomando aquí un 
concepto de la Pragmática integrada, es una partícula que hace que el contenido que le sigue posea mayor fuerza argumentativa que aquel al que no precede; en tercer lugar, se trata de un elemento que presenta el contenido en el que aparece como una conclusión contraria a otra que se pudiera inferir a partir del contenido que no precede. A nuestro modo de ver, si bien no se puede negar la justeza y la operatividad de estas instrucciones, estas resultan no obstante insuficientes para resolver el problema de cómo explicar el enunciado fallido. Ninguna de ellas puede responder a preguntas como estas: ¿por qué (2) no es pragmáticamente apropiado?; ¿por qué sí seria posible usar pero si modificáramos la secuencia que lo sigue (por ejemplo, diciendo: "No fue ayer cuando llegó el paquete, pero no pasa nada por haberles dado mal esa información”)?; ¿por qué el enunciado sería perfectamente apropiado si en lugar de pero introdujéramos sino como conjunción entre los dos contenidos relacionados ("No fue ayer cuando llegó el paquete, sino anteayer")?.

El problema de este enunciado está en que, al decir "No fue ayer cuando llegó el paquete, pero anteayer", un locutor está queriendo utilizar pero para "corregir" una información anterior. Ahora bien, como sabemos, pero no se puede utilizar en español para "corregir" un contenido previo, pues se trata de un conector que presenta una 'restricción' a una información anterior, no una 'corrección'. Para elucidar la diferencia "instruccional" entre las dos palabras, podriamos recuperar las etiquetas de 'restricción' y de 'corrección' $\mathrm{y}$, a partir de ellas, definir una instrucción procedimental, es decir, cognitiva. Ahora bien, proceder de este modo nos obliga en primera instancia a tener que salvar la difícil tarea de definir 'restricción' y 'corrección' de modo inequívoco y operativo, sin saber cómo salvar el escollo de no confundir, además, lengua y metalengua.

Hay otro camino para explicar la diferencia entre las dos palabras: se trata de elucidar cuáles son las instrucciones de la estructura profunda que deben subyacer en las frases donde se utilice cada una de ellas, instrucciones que darán al interlocutor la información que necesita para interpretar los enunciados donde pero y sino aparezcan. Para dar cuenta de esas instrucciones, el enfoque que queremos seguir nos lleva a proponer que no podemos estudiar pero y sino sin tener en cuenta los contextos lingüísticos donde se insertan esas dos palabras cuando aparecen en el discurso. Decir que sino es un conector que relaciona dos contenidos, haciendo que uno se presente como una 'corrección' con respecto de otro, nos obliga a definir tanto el contenido que se presenta como 'corrección' como aquel que se ve afectado por este. Ahora bien, no podremos dar cuenta de las características de estos contenidos partiendo del enunciado mismo donde se inserta el conector sino que deberemos partir de una frase que, como entidad abstracta, se convierta en objeto de análisis y cuyo estudio nos permita proveer una significación que, después, pueda explicar el sentido (o el sinsentido) del enunciado. Llamaremos a los contenidos del enunciado X e Y y, a sus correspondientes desde el nivel de la frase, $\mathrm{P}$ y Q. El enunciado, recordemos, 
es lo que es perceptible por el interlocutor; la frase, por el contrario, es lo que constituye el objeto de análisis del lingüista. Por ejemplo:

\begin{tabular}{|l|l|l|}
\hline Miembro del discurso 1 & \multicolumn{2}{|c|}{ Miembro del discurso 2. } \\
\hline ESTRUCTURA SUPERFICIAL (X) & \multirow{4}{*}{ SINO } & ESTRUCTURA SUPERFICIAL (Y) \\
\cline { 1 - 1 } No fue ayer cuando llegó el paquete & & Anteayer. \\
\cline { 1 - 1 } ESTRUCTURA PROFUNDA (P) & & ESTRUCTURA PROFUNDA (Q) \\
Ayer no llegó el paquete. & & Anteayer (llegó el paquete). \\
\hline
\end{tabular}

Cuadro 1: Representación de sino

Empecemos estudiando las características de P. Como es sabido, sino es un conector en español que tiene como alcance contenidos que están negados. Ahora bien, cualquier tipo de negación no es condición suficiente para poder utilizar sino en español. La negación del primer miembro del discurso ha de ser de tipo polémica, no descriptiva. En la Pragmática integrada, se considera que una negación es polémica si constituye "le rejet d'une affirmation préalable" (Ducrot, 1980: 28-29) de modo tal que "le locuteur s'oppose [...] à un énonciateur que sa propre énonciation met en scène" (Ducrot, 1984: 217; Anscombre, 1990: 93). Es descriptiva, por el contrario, una negación que "présente les faits [...] de première main, et qui n’a pas pour fonction immédiate de contredire" (Ducrot, 1980 : 29), es decir, la "pure représentation d'un état de fait" (Ducrot, 1984: 217; Anscombre, 1990: 93)6. La prueba de que sino exige que la negación sea del primer tipo y no del segundo es que si afecta a enunciados que constituyen una negación descriptiva da lugar a secuencias pragmáticamente inaceptables:

(5)\# "No estudié el examen, sino aprobé”.

El hecho de que sino aparezca afectando a contenidos que han de mostrar una negación de tipo polémico es algo estrechamente relacionado con su semantismo. Veamos por qué. Como se sabe desde los primeros escritos sobre polifonía atribuidos a la Pragmática integrada ${ }^{7}$, los enunciados que presentan una negación son polifónicos. Ello quiere decir que varias voces discursivas son responsables de diferentes puntos de vista presentes en ellos. Según mostraron Anscombre y Ducrot, en un enunciado negado hay, por un lado, un punto de vista que ha de atribuirse a la voz discursiva responsable del contenido no negado (p. e. "Estudié el examen") y, por otro lado, otro punto de vista atribuible a otra voz discursiva diferente que sería responsable de la marca de la negación (i. e. "No"). A nuestro entender, este reparto polifónico se da tanto en el caso de la negación polémica como en el

\footnotetext{
${ }^{6}$ Ducrot (1980) distingue entre negación descriptiva y negación polémica o metalingüistica, mientras que en Ducrot (1984) y en Anscombre (1990) se distinguen tres: descriptiva, polémica y metalingüistica, con variaciones en cuanto a los criterios con respecto de 1980 . El análisis polifónico que presentamos aquí parte de estas definiciones pero no se corresponde forzosamente con lo que estos autores describen para el francés.

7 Véase Ducrot (1980), Anscombre-Ducrot (1983), Ducrot (1984), Ascombre (1985), Anscombre-Ducrot (1986).
} 
de la negación descriptiva pero hay algo que las separa: en la negación polémica las dos voces responsables de los dos puntos de vista deben ser identificadas con dos locutores (en concreto, con un Exlocutor y un Locutor) mientras que en la negación descriptiva esas dos voces habrian de ser identificadas con un solo Locutor que convoca dos enunciadores (uno, el del contenido afirmativo y, otro, el responsable de la negación). Esto haría que la repartición polifónica de pero y de sino en el discurso fuera de este modo:

Negación descriptiva: No estudié el examen, pero aprobé.

\begin{tabular}{|c|c|c|}
\hline \multicolumn{3}{|c|}{ ESTRUCTURA SUPERFICIAL: No estudié el examen, pero aprobé. } \\
\hline \multicolumn{3}{|c|}{ ESTRUCTURA PROFUNDA } \\
\hline $\begin{array}{c}\text { Enuncia- } \\
\text { dor1 }\end{array}$ & Estudié el examen. \\
$\begin{array}{c}\text { Enuncia- } \\
\text { dor2 }\end{array}$ & No. & PERO Aprobé. \\
\hline \multicolumn{2}{|c|}{ El Locutor se identifica con los dos puntos de vista (E1 y E2). } \\
\hline
\end{tabular}

Cuadro 2: Negación descriptiva con pero

Negación polémica: No fue ayer cuando llegó el paquete, sino anteayer.

\begin{tabular}{|c|c|c|c|}
\hline \multicolumn{4}{|c|}{ ESTRUCTURA SUPERFICIAL: No fue ayer cuando llegó el paquete, sino anteayer } \\
\hline \multicolumn{4}{|c|}{ Locutor } \\
\hline $\begin{array}{l}\text { Exlocu- } \\
\text { tor }\end{array}$ & El paquete llegó ayer & \multirow[t]{2}{*}{ SINO } & \multirow{2}{*}{ Anteayer } \\
\hline Locutor & No. & & \\
\hline
\end{tabular}

Cuadro 3: Negación polémica con sino

Como vemos en los dos esquemas, en el caso de la negación descriptiva ("No estudié el examen, pero aprobé") el Locutor se identifica con los dos puntos de vista atribuidos a los dos enunciadores que convoca; sin embargo, en la negación polémica, el Locutor no se identifica con el punto de vista atribuido al Exlocutor. Esto es lo que permite la aparición de pero y sino en sendos ejemplos: si aceptamos que pero se antepone a un contenido que se presenta como una 'restricción' a un contenido anterior mientras que sino se antepone a un contenido que se presenta como una 'corrección', podemos justificarlo con la diferente repartición de las voces discursivas que surgen de los dos diferentes tipos de negación: un Locutor hará una 'restricción' a un contenido donde son presentados dos puntos de vista con los que se siente identificado, pero hará una 'corrección' sobre un contenido donde se presentan dos puntos de vista y uno de ellos es atribuido a una voz discursiva con la que el Locutor no se identifica. La 'restricción' de sino y la 'corrección' de pero se corresponden, por tanto, con una diferente repartición de voces discursivas como responsables de los puntos de vista que subyacen a los contenidos que se ven afectados por ambos conectores. A la vista de 
ese análisis, la aparición de pero y de sino en una frase atiende a las siguientes instrucciones:

Instrucciones de sino: Sea $\mathrm{P}$ una frase que constituye obligatoriamente una negación polémica cuyo contenido está convocado por dos voces discursivas: por un lado, un Exlocutor responsable de una aserción; por otro lado, el Locutor responsable de la negación de la aserción. El Locutor de la frase no se identifica con la aserción del Exlocutor.

Instrucciones de pero: Sea $\mathrm{P}$ una frase que, de constituir una negación, esta es de naturaleza descriptiva. Sea el contenido de P convocado por un Locutor el cual, en el caso de convocar dos Enunciadores responsables de dos puntos de vista, se identifica con los dos puntos de vista.

La definición de estas instrucciones sí nos permite ahora saber por qué (2) resulta afectado:

(2) \# "No fue ayer cuando llegó el paquete, pero anteayer".

Como ahora sabemos, si pero afecta a un contenido previo que constituye una negación, esta debe ser de naturaleza descriptiva, no polémica. Como hemos mostrado, una negación como "No fue ayer cuando llegó el paquete" es de naturaleza polémica, lo cual impide al interlocutor seguir las instrucciones para hallarle un sentido al enunciado.

Podemos plantear un procedimiento similar al anterior para analizar la inaceptabilidad de un enunciado como (3). El DPDE define en efecto como una partícula que "[p]resenta el miembro del discurso al que afecta como una confirmación deliberada de lo expresado antes o de lo supuesto a partir del contexto, o como una prueba de ello. Se proporciona así un mayor grado de validez o de fuerza argumentativa al contenido transmitido, sobre el que ahora se insiste.” (Llopis Cardona, DPDE). ¿Por qué en (4) la interpretación de en efecto como un signo que confirma lo anterior resulta afectada?; ¿por qué no podemos interpretar aquí que el segundo miembro del discurso se presenta como una prueba para avalar lo dicho en el primer miembro? La respuesta a estas cuestiones tiene que ver de nuevo con que el marcador actúa en un contexto lingüístico que debe cumplir ciertas restricciones.

En Piedehierro (2012) señalamos que en efecto, cuando actúa como conector, puede vehicular los siguientes sentidos: confirmación, demostración, ilustración, justificación, y consecuencia. Estos, determinados a priori como hipótesis externas, son definidos gracias a que en cada caso en efecto aparece en el discurso convocando la aparición de diferentes restricciones semántico-pragmáticas (hipótesis internas), las cuales afectan de forma diferente a los contextos lingüísticos donde aparece el conector. Hay, no obstante, una restricción que parece cumplirse para todos los sentidos vehiculados por en efecto: este debe tener siempre como alcance lo que Palmer (2001 [1986]) denomina un contenido REALIS, es decir, no modalizado. Esta idea ha sido señalada en varios trabajos, donde se sostiene que en efecto 
actúa sobre aserciones (o proposiciones), reforzándolas (Barrenechea, 1979; Martín Zorraquino y Portolés, 1999) y que ese refuerzo hace que la aserción sea afirmada (Casado Velarde, 1998; Santos Río, 2003), explicada o ampliada (Martín Zorraquino y Portolés, 1999) y/o, sobre todo, confirmada (Moliner, 2007; Calsamiglia y Tusón, 1999; Fuentes, 1994; Martín Zorraquino y Portolés, 1999; Santos Río, 2003; Falk, 2006; Llopis, 2011). Ahora bien, lo que no se explica en la bibliografia sobre el marcador es cómo es posible que en efecto actúe afectando a aserciones (contenidos REALIS) y, al mismo tiempo, la afirmación que lo precede tenga la particularidad de presentarse como pretendidamente discutible, como susceptible de discusión (Fuentes, 1994) o como una "conjetura" (Falk, 2006), es decir, como contenidos, según la denominación de Palmer (2001 [1986]) IRREALIS. En Piedehierro (2012), el recurso a la división entre estructura superficial y estructura profunda nos sirvió para dar cuenta de este hecho. Observemos por ejemplo (6):

(6) "Posiblemente, una de las aportaciones más importantes de esta teoría a la investigación lingüística sea la revisión y la redefinición del concepto de contexto. En efecto, frente a la idea de contexto manejada hasta el momento, la Teoría de la Relevancia postula una concepción cognitiva del contexto". (E. Montolío, 1998)

En este ejemplo, en efecto vehicula (es nuestra hipótesis externa) un sentido de demostración (Piedehierro, 2012), es decir, que el hablante al utilizar el marcador está queriendo demostrar, en el segundo miembro del discurso, la verdad de una aserción contenida en el primer miembro. Tenemos aquí precisamente un buen ejemplo del problema que evocábamos antes: la presencia del adverbio "posiblemente" impone una modalidad epistémica al contenido que se ve afectado por el conector. Como consecuencia, ese contenido no es una aserción o, al menos, no lo es en el sentido lógico (formal) en que se entiende el concepto. ¿Cómo explicar entonces la restricción de en efecto que evocábamos más arriba? Con el recurso a la división entre estructura superficial y estructura profunda:

\begin{tabular}{|c|c|c|}
\hline Miembro del discurso1 & \multicolumn{2}{|c|}{ Miembro del discurso2 } \\
\hline X (ESTRUCTURA SUPERFICIAL) & \multirow{4}{*}{$\begin{array}{l}\text { EN } \\
\text { EFECTO }\end{array}$} & Y (ESTRUCTURA SUPERFICIAL) \\
\hline $\begin{array}{l}\text { "Posiblemente, una de las aporta- } \\
\text { ciones más importantes de esta } \\
\text { teoría a la investigación lingüís- } \\
\text { tica sea la revisión y la redefini- } \\
\text { ción del concepto de contexto." }\end{array}$ & & $\begin{array}{l}\text { "frente a la idea de contexto manejada } \\
\text { hasta el momento [...] la Teoria de la } \\
\text { Relevancia postula una concepción } \\
\text { cognitiva del contexto" }\end{array}$ \\
\hline P (ESTRUCTURA PROFUNDA) & & Q (ESTRUCTURA PROFUNDA) \\
\hline $\begin{array}{l}\text { Esta teoría revisa y redefine el } \\
\text { concepto de contexto }\end{array}$ & & $\begin{array}{l}\text { La Teoría de la Relevancia postula una } \\
\text { concepción cognitiva del contexto }\end{array}$ \\
\hline
\end{tabular}

Cuadro 4: Representación de en efecto

Como se puede observar, en la estructura superficial aparece X, contenido relativo al nivel del enunciado, el segmento perceptible que aparece en 
el contexto izquierdo del conector. Se trata de un enunciado declarativo (desde el punto de vista de la enunciación) pero que presenta una modalidad IRREALIS, en concreto, proposicional de tipo epistémica especulativa ("Posiblemente..."). En la estructura profunda, por su parte, aparece P, relativo al nivel de la frase, contenido que representa el segmento que realmente sufre el alcance del marcador. P es una auténtica aserción, es decir, es un contenido REALIS, no modalizado. Como vemos, gracias a tener en cuenta esta división entre estructura superficial y estructura profunda, podemos explicar cómo es posible que en efecto tenga como alcance aserciones y, sin embargo, pueda verse precedido por enunciados modalizados epistémicamente. Pero es que este recurso nos va a servir igualmente para explicar la inaceptabilidad de (3):

(3) \# "Tienes que irte; en efecto es muy peligroso que te quedes".

Como vemos en este ejemplo, el contenido del primer miembro del discurso (X), está representado por un enunciado IRREALIS, pues ostenta una modalidad deóntica. A partir de un enunciado con estas características, es imposible hallar en la estructura profunda una posible aserción que constituya el eventual alcance de en efecto, pues la deóntica es una modalidad relacionada con eventos que no están actualizados, que están en potencia. Por el contrario, la modalidad proposicional del contenido X de (6) sí permite recuperar una aserción en la estructura profunda, pues esta modalidad, recordemos, está relacionada con la actitud del hablante hacia el valor de verdad del estatus factual de la proposición:

Instrucciones de en efecto: Sea $\mathrm{P}$ una frase que constituye obligatoriamente un contenido REALIS, es decir, un contenido no modalizado, una aserción.

Terminemos comentando la inaceptabilidad de (4). Pues ha sido descrita como una partícula que puede tener dos funciones fundamentales ${ }^{8}$ : por un lado, puede funcionar como una conjunción introductora de subordinadas causales ("Por lo demás, esto parece Londres, pues lleva por lo menos quince dias lloviendo", Miche, 1994) y, por otro, como una partícula "ilativa" que tiene dos usos, uno tónico, donde aparece en posición incidental en el enunciado expresando un matiz consecutivo ("Paco no ha leído el libro. No le des, pues, la lata con tus explicaciones", Miche, 1994) y otro átono, típicamente denominado empleo "continuativo" con sus variantes de "réplica" ("A : Voy al centro. B : Pues acaba de irse el autobús", Portolés, 1989), de "respuesta"

${ }^{8}$ Véase, por ejemplo: Gramática de la Lengua Española $(1931 ; \S 345)$, Esbozo (1973; § 3.22.2 y 3.22.3), Nueva Gramática (2009-2011: § 46.3; 46.6; § 46.11; § 30.13j y ss.; § 47.1; 47.9), Alcina y Blecua (1975), Mariner Bigorra (1981), Portolés (1989, 2001 [1998]), Miche (1994), Sicot-Domínguez (1998), Chevalier y Molho (1986), Martín Zorraquino y Portolés (1999), Iglesias Recuero (2000). 
(“A: ¿Qué hora es? B: Pues ni idea.") y "adversativa” ("Que no querías sopa, pues toma tres tazas"). El problema de la inaceptabilidad de (4) es que al decir "\#Juan terminó el trabajo, pues se fue", la partícula pues funciona estableciendo un orden secuencial entre dos acontecimientos que se suceden, dentro de un ordenamiento de hechos: primero, "Juan terminó el trabajo"; segundo, "Juan se fue". Este funcionamiento estaba presente en el étimo latino de la partícula, la voz POST, que podía expresar un sentido tanto locativo como espacial ('detrás', 'después',...). Ahora bien, en su proceso de evolución durante la Edad Media, pues dejó de expresar una posterioridad temporal y pasó a especializarse en una forma que iba a vehicular una posterioridad de otra naturaleza, llegando al nivel de la enunciación (Álvarez-Ejzenberg y Piedehierro, en prensa). La idea de que pues afecta a la enunciación y no al enunciado ha sido puesta de manifiesto en varios trabajos que han estudiado la partícula (Chevalier y Molho 1986; Portolés, 1989; Miche, 1994; Sicot-Dominguez, 1998; RAE y AALE, 2009-2011). En consonancia con esta idea, en Álvarez-Ejzenberg y Piedehierro (en prensa) definimos a pues como un "ordenador dialógico de enunciaciones", lo cual significa que se trata de una partícula que recrea un esquema en el discurso que implica la puesta en escena de dos voces discursivas responsables de dos puntos de vista diferentes que, además, dan lugar a dos enunciaciones diferentes. Esta descripción nos permitiría dar cuenta de la interpretación de pues en un ejemplo como (7):

(7) "Parece claro que nos dirigimos hacia la repetición de unas nuevas elecciones, pues los representantes de los partidos politicos no consiguen ponerse de acuerdo para formar gobierno".

En un enunciado como (7), pues sería descrito como una conjunción causal. Ahora bien, lo que introduce pues no es una causa que justifique un hecho explicitado en el primer miembro del discurso, sino una explicación a por qué el locutor ha enunciado el contenido que aparece a la izquierda del marcador. El alcance de pues no es X, no es el enunciado del primer miembro del discurso, sino la enunciación misma de $\mathrm{X}$, el hecho de haberlo dicho:

\begin{tabular}{|l|l|l|}
\hline Miembro del discurso1 & Miembro del discurso2 \\
\hline X (ESTRUCTURA SUPERFICIAL) & \multirow{2}{*}{$\begin{array}{l}\text { PUES } \\
\text { "Parece claro que nos dirigimos } \\
\text { hacia la repetición de unas nue- } \\
\text { vas elecciones" }\end{array}$} & $\begin{array}{l}\text { "los representantes de los partidos polí- } \\
\text { ticos no consiguen ponerse de acuerdo } \\
\text { para formar gobierno" }\end{array}$ \\
$\begin{array}{l}\text { P (ESTRUCTURA PROFUNDA) } \\
\text { Digo que [parece claro que nos di- } \\
\text { rigimos hacia la repetición de } \\
\text { unas nuevas elecciones] }\end{array}$ & \multirow{5}{*}{$\begin{array}{l}\text { Voy a decir que [los representantes de } \\
\text { los partidos politicos no consiguen po- } \\
\text { nerse de acuerdo para formar gobierno] }\end{array}$} \\
\hline
\end{tabular}


De este modo, la interpretación de (7) podría ser glosada como: [Digo que $\mathrm{X}$ porque a continuación voy a decir que Y]: [Digo que parece claro que nos dirigimos hacia la repetición de unas nuevas elecciones porque a continuación voy a decir que los representantes de los partidos políticos no consiguen ponerse de acuerdo para formar gobierno].

Lo que hace un locutor que emite un enunciado como (7) es convocar dos voces discursivas que son responsables de dos enunciaciones diferentes. Pues se encarga de ordenarlas, es decir, de ponerlas en el discurso, una detrás de la otra. De este modo, mantiene su instrucción primigenia heredada de la voz latina POST, dado que sigue expresando una posterioridad. Ahora bien, esta posterioridad deja de expresarse a nivel del enunciado y pasa a expresarse a nivel de la enunciación, lo que explica que la interpretación de un ejemplo como (4) resulte afectada, dado que aquí es imposible establecer que el alcance del marcador sea otra cosa diferente del contenido enunciado: [\# Digo que Juan terminó el trabajo porque a continuación voy a decir que Juan se fue]. Ello es así porque en (4) no es posible recuperar la instrucción que impone pues en su funcionamiento discursivo:

Instrucción de pues: sea $\mathrm{P}$, el contenido que constituye el alcance del marcador, la enunciación del locutor, y no el enunciado.

En el análisis que acabamos de presentar, hemos intentado hacer explícitas algunas de las ideas de la Pragmática integrada que, a nuestro juicio, no han sido suficientemente desarrolladas en los estudios de los marcadores del discurso en el ámbito hispánico (como el par estructura superficial-estructura profunda o el par sentido-significación). Por otro lado, hemos pretendido mostrar que el concepto de instrucción tal como se entiende en los trabajos de Anscombre y Ducrot está en realidad muy alejado de una concepción cognitiva de la interpretación semántica como es la de la Teoría de la relevancia. Si para esta las instrucciones conciernen al procesamiento de los signos, para la Pragmática integrada las instrucciones conciernen más bien a lo que podríamos considerar su funcionamiento. Esta concepción instruccional recuperada de la Pragmática integrada nos ha permitido, finalmente, explicar por qué ciertos enunciados resultan pragmáticamente afectados, ciñéndonos al estudio de las restricciones que los marcadores imponen, a su izquierda y a su derecha, cuando aparecen en el discurso.

\section{Conclusiones}

En este trabajo hemos querido dar cuenta de las diferencias que separan a dos teorias clásicas como son la Teoría de la relevancia y la Pragmática integrada en lo que concierne a la conceptualización de ciertos constructos utilizados por ambas, como son el par frase-enunciado y el concepto de instrucción. Esta exposición tenía como objetivo subrayar el hecho de que es necesario tener en cuenta las divergencias que existen entre un enfoque de 
tipo cognitivo y otro de tipo lingüístico a la hora de enfrentarse al análisis de la interpretación de los enunciados. Hemos mostrado en concreto que, si para la pragmática relevantista la interpretación es fruto de un proceso inferencial que tiene lugar gracias a la mediación entre los signos y el contexto; para la Pragmática integrada, por el contrario, la interpretación está anclada en las estructuras lingüísticas mismas.

Como hemos explicado, esta diferencia está relacionada con el hecho de que las dos perspectivas responden en realidad a dos preguntas diferentes: mientras la pragmática de Sperber y Wilson busca saber qué significado entendemos a partir de un enunciado, la de Anscombre y Ducrot trata de averiguar qué características tiene un enunciado dado para poder significar lo que significa. Dicha diferencia podría ilustrarse con la que media entre preguntarse qué quiere decir un hablante cuando nos dice: "No tomo azúcar", es decir, si quiere simplemente constatar un hecho, avisarnos de que no debemos añadir azúcar a su café o disculparse por rechazar un dulce que acabamos de ofrecerle, y preguntarse qué peculiaridades contiene la cadena hablada <No tomo azúcar $>$ que explican que pueda ser interpretada como una constatación, un aviso o una disculpa.

En la segunda parte del artículo hemos pretendido mostrar que solamente un enfoque de análisis que parta del estudio de las restricciones que los signos imponen a su aparición en el discurso puede ser válido para analizar secuencias pragmáticamente afectadas donde aparecen marcadores del discurso. Ello es así porque este enfoque se basa en la tesis de que la interpretación no depende de las situaciones en las que tiene lugar un discurso particular, sino de peculiaridades intrínsecas al funcionamiento lingüístico. Nuestro análisis se ha servido de la división que la Pragmática integrada establece entre estructura superficial y estructura profunda y hemos intentado partir del concepto instrucción tal como se entiende en esta teoría, esto es, como una consigna lingüística acatada por una palabra que, junto con otras palabras, conforma una frase de una lengua, comprometiéndola a ser susceptible de mostrar una determinada significación y, como consecuencia, de explicar el sentido de un enunciado.

\section{REFERENCIAS}

ALCINA FRANCH, J. \& J. M. BLECUA (1975): Gramática española, Barcelona: Ariel.

ÁLVAREZ-EJZENBERG, F. \& PIEDEHIERRO, C. (en prensa): "Pues vs. puis: la posterioridad de la enunciación frente a la posterioridad del enunciado. Estudio de las partículas en los primeros textos en lengua francesa y española". Actes du XV Colloque international de linguistique ibéro-romane, Rouen 2015.

ANSCOMBRE, J. C. (1985) : "Mention, citativité et autres blablas", Langages, 80, pp. 9-34.

ANSCOMBRE, J. C. (1990) : "Thème, espaces discursifs et représentation événementielle", Anscombre, J. C. \& Zaccaria, G (eds.): Fonctionnalisme et pragmatique, Milan: Unicopli, pp. 43-150. 
ANSCOMBRE, J. C. \& DUCROT, O. (1978) : "Lois logiques et lois argumentatives", Le français moderne, 46, pp. 347-357.

ANSCOMBRE, J. C. \& DUCROT, O. (1983): L'argumentation dans la langue, Liège : Mardaga.

ANSCOMBRE, J. C. \& DUCROT, O. (1986): "Argumentativité et informativité", Meyer, M. (ed.) : De la métaphysique à la rhétorique, Bruxelles: Université de Bruxelles, p. 7994.

BARRENECHEA, A. M. (1979): “Operadores pragmáticos de actitud oracional: los adverbios en mente y otros signos", VVAA (ed.): Estudios lingüisticos y dialectológicos. Temas hispánicos. Argentina: Hachette, pp. 39-59.

BLAKEMORE, D. (1987): Semantic constraints on relevance, $\mathrm{Ox}-$ ford: Blackwell.

BLAKEMORE, D. (1992): Understanding utterances, Oxford: Blackwell.

BLAKEMORE, D. (2002): Relevance and Linguistic Meaning. The Semantics and Pragmatics of Discourse Markers, Cambridge: Cambridge University Press.

BRIZ, A., PONS, S. \& Portolés, J. (coords.) (2008): Diccionario de particulas discursivas del espanol. En linea, www.dpde.es

CASADO VELARDE, M. (1998): "Lingüística del texto y marcadores del discurso", Martín Zorraquino, M. A. y Montolío Durán, E.: Los marcadores del discurso. Teoría y análisis, Madrid: Arco/ Libros, pp. 55-70.

CHEVALIER, J. C. \& MOLHO, M. (1986): "De l'implication: esp. pues, fr. puis", Travaux de Linguistique et de Littérature XXIV, 1, pp. 23-34.

CORTÉS, L. \& CAMACHO, M. (2005): Unidades de segmentación $y$ marcadores del discurso, Madrid: Arco/Libros.
DUCROT, O (1980) : Les échelles argumentatives, Paris : Minuit.

DUCROT, O. (1984) : Le dire et le dit, Paris: Les éditions de minuit.

DUCROT, O. (1986 [1984]): El decir y lo dicho. Polifonía de la enunciación, Barcelona: Paidós.

DUCROT, O. (1991 [1972]) : Dire et ne pas dire. Principes de sémantique linguistique, Paris : Hermann.

DUCROT, O. et al. (1980) : Les mots du discours, Paris : Minuit.

FALK, J. (2006): “En efecto, es su cumpleaños mañana. Observaciones sobre el marcador del discurso en efecto", VV.AA.: Discurso, interacción e identidad. Homenaje a Lars Fant, Stockholms Universitet, pp. 37-63.

FUENTES RODRÍGUEZ, C. (1994): "Usos discursivos y orientación argumentativa: de hecho, en efecto, efectivamente", Español Actual, 62, pp. 5-18.

GRICE, H. P. (1975): "Logic and conversation", Cole, P. y Morgan, J.: Syntax and Semantics 3, Speech Acts. New Cork: Academic Press. Traducción española: "Lógica y conversación", Valdés Villanueva (ed.): La búsqueda del significado, Tecnos, 1991, pp. 511-530.

IGLESIAS RECUERO, S. (2000): "La evolución histórica de pues como marcador discursivo hasta el siglo XV", BRAE. Cuaderno CCLXXX, pp. 209-307.

LLOPIS CARDONA, A. B. (2011): Las funciones de los marcadores discursivos a través del análisis de "eso sí", "en este sentido", "en efecto" y “نverdad?”. Tesi doctoral. Universitat de València. Facultat de Filologia, Traduccio i Comunicació. Departament de Filologia Espanyola.

LOUREDA LAMAS, Ó. \& ACÍN VILLA, E. (coords.) (2010): Los estudios sobre marcadores del discurso 
en español, hoy, Madrid: Arco/Libros.

MARINER BIGORRA, S. (1981): "Pues y doncs adversativos", Logós Semantikós, Studia Lingüistica in Honores Eugenio Coseriu, vol. IV, Madrid: Gredos, pp. 289297.

MARTÍN ZORRAQUINO, M ${ }^{a}$ A. \& PORTOLÉS LÁZARO, J. (1999): "Los marcadores del discurso", Bosque, I. y Demonte, V. (coords.): Gramática descriptiva de lengua española, vol. 3: Entre la oración y el discurso. Morfología. Madrid, Espasa, Cap. 63, pp. 4050-4213.

MICHE, E. (1994): "Description sémantico-pragmatique de la marque espagnole pues", Cahiers de linguistique française, 15, 51-76.

MOLINER, M. (2007 [1966-1967[): Diccionario de uso del español. Madrid, Gredos, $3^{\mathrm{a}}$ ed.

MURILLO ORNAT, S. (2010): "Los marcadores del discurso y su semántica", Loureda Lamas, O. y Acín Villa, E. (coords.), pp. 183-240.

PALMER, F. R. (2001 [1986]): Mood and Modality, Cambridge: Cambridge University Press.

PIEDEHIERRO, C. (2012) : Un modèle de pragmatique linguistique pour l'analyse des marqueurs $d u$ discours. Application à l'étude contrastive de "en efecto" et "en effet". Thèse de Doctorat. Tesis de Doctorado. (EHESS, Paris; UAM, Madrid)

PONS BORDERÍA, S. (2004): Conceptos y aplicaciones de la Teoría de la Relevancia, Madrid: Arco/Libros.

PORTOLÉS, J. (1989): "El conector argumentativo pues", Dicenda, 8, pp. 117-133.

PORTOLÉS, J. (1993): "La distinción entre los conectores y otros marcadores del discurso en español”, Verba, 20, pp. 141-170.

PORTOLÉS, J. (1994): "Pertinencia y pragmática", Revista de Occidente, 154, 55-66.

PORTOLÉS, J. (1995a): "Diferencias gramaticales y pragmáticas entre los conectores discursivos pero, sin embargo y no obstante", Boletín de la Real Academia Española, 75, pp. 231-269.

PORTOLÉS, J. (1995b): "Del discurso oral a la gramática: la sistematización de los marcadores discursivos", en Cortés Rodríguez, L. (ed.): Actas del I Simposio sobre análisis del discurso oral, Almeria: Universidad de Almería, pp. 141-171.

PORTOLÉS, J. (1998a): "El concepto de suficiencia argumentativa", Signo y Seña, 9, pp. 199-224.

PORTOLÉS, J. (1998b): “Dos pares de marcadores del discurso: en cambio y por el contrario, en cualquier caso y en todo caso, en Martín Zorraquino, $\mathrm{M}^{\mathrm{a}}$ A. \& Montolío Durán, E. (coors.): Los marcadores discursivos. Teoría y análisis. Madrid: Arco/Libros, 243-264.

PORTOLÉS, J. (1999): "Los ordenadores del discurso y el lenguaje periodístico", Garrido Medina, J. (ed): La lengua y los medios de comunicación, I, Madrid: Universidad Complutense de Madrid, pp. 161-169.

PORTOLÉS, J. (2000a): "Dos perspectivas en el estudio de los marcadores discursivos", De Miguel, E., M. Fernández Lagunilla y F. Cartoni (eds.): Sobre el lenguaje: miradas plurales $y$ singulares, Madrid: Arrecife, pp. 101-119.

PORTOLÉS, J. (2000b): “El significado informativo de los marcadores del discurso", Bustos Tovar, J., Charandeau, P., Girón Alconchel, J. L., Iglesias Recuero y 
López Alonso, I. (eds): Lengua, discurso, texto (I Simposio Internacional de Análisis del Discurso), I, Madrid: Visor, pp. 683-692.

PORTOLÉS, J. (2001 [1998]): Marcadores del discurso, Barcelona: Ariel.

PORTOLÉS, J. (2004a) Pragmática para hispanistas, Madrid: Sintesis.

PORTOLÉS, J. (2004b): "Consideraciones metodológicas para el estudio del significado de los marcadores del discurso", Narvaja de Arnoux, E. y García Negroni, M. (eds.): Homenaje a Oswald Ducrot, Buenos Aires: Eudeba, pp. 315-336.

RAE (1931): Gramática de la lengua española, Madrid: Espasa Calpe.

RAE (1973): Esbozo de una nueva gramática de la Lengua Española, Madrid: Espasa Calpe.

RAE \& AALE (2009-2011): Nueva gramática de la lengua española, Madrid: Espasa.
SANTOS RÍO, L. (2003): Diccionario de particulas. Salamanca: LusoEspañola de Ediciones.

SICOT-DOMÍNGUEZ, S. (1998): "Le relateur pues : du signifiant à la fonction", LUQUET, G., (éd.) : Travaux de linguistique hispanique. Actes du VIIe Colloque de linguistique hispanique, Paris: Presses de la Sorbonne Nouvelle, pp. 169 183.

SPERBER, D. \& WILSON D. (1989 [1986]): La pertinence. Communication et congnition. Paris : Les éditions de minuit.

WILSON, D. \& SPERBER, D. (1990): "Forme linguistique et pertinence", Cahiers de Linguistique Française, 11, pp. 13-48 ; en inglés: (1993): "Linguistic form and Relevance", Lingua, 90, pp- 125. 